\title{
Cosmic-ray research with AMS-02 on the Inter- national Space Station
}

H. Gast for the AMS Collaboration

I. Physikalisches Institut B, RWTH Aachen University

DOI: http://dx.doi.org/10.3204/DESY-PROC-2013-04/gast_henning

Henning Gast gave a talk on new results of the AMS-02 detector onboard the ISS. Three weeks after the Patras Workshop in Mainz, updated AMS results were presented at the 33rd International Cosmic Ray Conference (ICRC) and are described in the corresponding proceedings in detail.

For convenience of the Patras participants, the editors collected the following links to the respective AMS publications in addition to the slides that are available on the conference website.

\section{References}

[1] S. Haino et al., "Precision measurement of the proton flux with AMS," https://143.107.180.38/indico/contributionDisplay $\cdot$ py?sessionId=3\&contribId=1265\&conf Id=0.

[2] V. Choutko et al., "Precision measurement of the helium flux with AMS," https: //143.107.180.38/indico/contributionDisplay . py?sessionId=3\&contribId=1262\&conf $I d=0$.

[3] A. Kounine et al., "Precision measurement of the positron fraction with AMS," https://143.107.180.38/indico/contributionDisplay $\cdot$ py?sessionId=3\&contribId=1264\&conf Id=0.

[4] S. Schael et al., "Precision measurements of the electron spectrum and the positron spectrum with AMS," https://143.107.180.38/indico/contributionDisplay . py?sessionId=3\&contribId=1257\& conf Id=0.

[5] B. Bertucci et al., "Precision measurement of the electron plus positron spectrum with AMS," https://143.107.180.38/indico/contributionDisplay $\cdot$ py?sessionId=3\&contribId=1267\&conf Id=0.

[6] A. Oliva et al., "Precision measurement of the boron to carbon ratio and nuclei with AMS," https://143.107.180.38/indico/contributionDisplay $\cdot$ py?sessionId=3\&contribId=1266\&conf Id=0.

[7] J. Casaus et al., "Determination of anisotropy with AMS," https: //143.107.180.38/indico/contributionDisplay. py?contribId=1261\&conf Id=0\&sessionId=3. 